\title{
The Solar Origin of Particle Events Measured by Parker Solar Probe
}

\author{
Athanasios Kouloumvakos ${ }^{1}$, Angelos Vourlidas ${ }^{2}$ (1) , Alexis P. Rouillard ${ }^{1}$, Edmond C. Roelof ${ }^{2}$, Rick Leske ${ }^{3}$, Rui Pinto ${ }^{1}$ (), and \\ Nicolas Poirier ${ }^{1}$ (i) \\ ${ }^{1}$ IRAP, Université Toulouse III—Paul Sabatier, CNRS, CNES, Toulouse, France; akouloumvakos@irap.omp.eu \\ 2 Johns Hopkins University Applied Physics Laboratory, Laurel, MD 20723, USA \\ ${ }^{3}$ California Institute of Technology, Pasadena, CA 91125, USA \\ Received 2020 April 29; revised 2020 June 24; accepted 2020 July 8; published 2020 August 18
}

\begin{abstract}
During the second solar encounter phase of Parker Solar Probe (PSP), two small solar energetic particle (SEP) events were observed by the Integrated Science Investigation of the Sun, on 2019 April 2 and 4. At the time, PSP was approaching its second perihelion at a distance of $\sim 24.8$ million kilometers from the solar center, it was in near-radial alignment with STEREO-A and in quadrature with Earth. During the two SEP events multiple narrow ejections and a streamer-blowout coronal mass ejection (SBO-CME) originated from a solar region situated eastward of PSP. We analyze remote-sensing observations of the solar corona, and model the different eruptions and how PSP was connected magnetically to the solar atmosphere to determine the possible origin of the two SEP events. We find that the SEP event on April 2 was associated with the two homologous ejections from active region 12738 that included two surges and EUV waves occurring in quick succession. The EUV waves appear to merge and were fast enough to form a shock in the low corona. We show that the April 4 SEP event originates in the SBO-CME. Our modeling work suggests that formation of a weak shock is likely for this CME.
\end{abstract}

Unified Astronomy Thesaurus concepts: Solar coronal mass ejections (310); Solar coronal mass ejection shocks (1997); Solar energetic particles (1491); Solar particle emission (1517)

Supporting material: animations

\section{Introduction}

Two decades of continuous measurements of solar energetic particles (SEPs) and simultaneous remote-sensing observations of the solar corona have significantly improved our understanding of the origin of energetic particles and their distribution in the heliosphere. Two dominant acceleration sites are involved in the generation of SEP events (see reviews by Desai \& Giacalone 2016; Reames 2017; Vlahos et al. 2019, and references therein). One occurs in solar flares and the other in shocks driven by coronal mass ejections (CMEs) in the corona and interplanetary medium. The largest and most intense SEP events are usually associated with fast and wide CMEs (e.g., Gopalswamy et al. 2004; Kahler \& Vourlidas 2013) and strong shock waves (e.g., Rouillard et al. 2016; Kouloumvakos et al. 2019). At events that are associated with very energetic CMEs and shock waves, the particles are continually accelerated in the solar atmosphere and can be released to distant magnetically connected spacecraft, far from the region where the flare occurred.

Multi-vantage point remote-sensing observations and in situ measurements from the twin STEREO spacecraft (Kaiser et al. 2008) shed light on the broad longitudinal extent of SEPs, including on the physical mechanisms responsible for the production of large, intense, and widespread SEP events at 1 au (e.g., Rouillard et al. 2012; Kouloumvakos et al. 2016; Lario et al. 2016, 2017). The expansion of shock waves low in the solar corona (EUV waves) seems to play a role in the production and release of SEPs (e.g., Kozarev et al. 2015; Park et al. 2015) but maybe not always (Lario et al. 2017). The case is much clearer higher in the solar corona (e.g., Rouillard et al. 2012; Kwon \& Vourlidas 2018) and in interplanetary space. Multi-viewpoint observations and reconstructions of shock waves suggest that those transients extend over a wide longitudinal range in the corona and can be responsible for the release of SEPs on magnetic field lines that are connected on the flanks of the CME shock. Additionally, state-of-the-art shock modeling and methods developed to infer shock parameters from remote-sensing observations proved to be essential to further understand the 3D properties of the expanding shock waves (Kozarev et al. 2015; Rouillard et al. 2016; Plotnikov et al. 2017; Kwon \& Vourlidas 2018). Shocks are supercritical over a wide extent and are capable of accelerating particles event at distant locations away from the eruption site. A connection between the properties of modeled shock waves and SEP characteristics has recently been established by Kouloumvakos et al. (2019).

Still, several questions remain open, mainly due to the difficulties in disentangling SEP acceleration from transport processes with 1 au observations only (e.g., Dalla et al. 2013; Laitinen et al. 2013). Particle transport mechanisms (including cross-field diffusion) may affect the timing and the characteristics of SEPs. This is a particular issue for small SEP events whose particle fluxes rise very gradually and remain close to the background fluxes for hours (e.g., Kouloumvakos et al. 2019). For some large SEP events, on the other hand, it has been difficult to determine the relative contribution of flares versus the CME shock waves in the acceleration and release process. One other aspect is that multiple SEP injections during the events are not clearly resolved from measurements near $1 \mathrm{au}$.

Past measurements made by Helios spacecraft showed that SEP events near the Sun are much less affected by interplanetary transport effects, with scattering and diffusion processes becoming less important. Now, the Parker Solar Probe (PSP: Fox et al. 2016) is making SEP measurements much closer to the Sun than ever before (Howard et al. 2019; 
McComas et al. 2019). We expect that they will improve our understanding of the origin and variability of SEP properties.

The purpose of this paper is to explore this new observational capability via the first detailed analysis of the solar origin of two small SEP events detected by PSP on 2019 April 2 (E. C. Roelof et al. 2020, in preparation) and April 4 (Leske et al. 2020) that originate from eruptions in the same solar region but have wildly different characteristics. We start our analysis by summarizing the observations in early 2019 April (Section 3). In Section 4, we investigate the magnetic connectivity of PSP, we perform detailed 3D modeling of the eruption associated with the SEP event of April 2, and we examine the possibility for shock formation in other eruptions and connect the modeling with the SEP measurements. Finally, we examine the origin of the April 4 SEP event. In Section 6 we summarize the results and conclude about the solar origin of the SEP events during the second PSP encounter.

\section{Orbital Details during Second Encounter}

PSP began its second solar encounter on 2019 March 30, and it lasted until April 10. The perihelion occurred on April 4 at a distance of $35.66 R_{\odot}(\sim 24.8$ million kilometers $)$ from center of the Sun. Figure 1(a) presents a view of the ecliptic from solar north between 2019 April 2 and 4. The orbital details are given in Carrington coordinates (Sun corotating frame). In this coordinate system the orbit of PSP executes a loop associated with the corotation and super-rotation of the spacecraft near perihelion. STEREO-A (STA) and Solar and Heliospheric Observatory (SOHO) spacecraft are observing the Sun from $\sim 1$ au in near-quadrature. The longitudinal separation between STA and Earth was $\sim 97^{\circ} .5$ on April 2. PSP and STA were almost radially aligned on April 2 with an angular separation of $\sim 2^{\circ}$, while PSP was separated from Earth by $\sim 95^{\circ}$. On April 4 PSP was located $\sim 33^{\circ}$ west of STA and $\sim 65^{\circ}$ east of Earth.

Observations from STA and near Earth provide good coverage of the low coronal activity below the in situ measurements of PSP. In Figure 1(a) we show the field of view (FOV) of the STA and LASCO coronagraphs and of the PSP WISPR-I (inner) heliospheric imager. The two WISPR imagers were observing the solar corona over STA's western limb. A CME eruption was observed by both STA/COR2 and WISPR on April 2 (see Section 3.3).

An estimation of the magnetic connection between the Sun and PSP is essential to determine where to focus our investigation for the solar sources of the SEP events measured in situ at the spacecraft. We start from a simplistic calculation of the magnetic connection by considering Parker spirals that connect PSP to the solar surface. For the calculation of the Parker spirals we use an average background solar wind speed of $350 \mathrm{~km} \mathrm{~s}^{-1}$ based on the in situ measurements made by PSP near perihelion. In Figure 1(b) we show a zoomed-in view of the orbit of PSP during the encounter and its position on April 2 and 4. We overplot two Parker spirals and the locus of all the spirals that magnetically connect PSP with the solar surface from April 2 to 4 . The footpoints of the spirals range from $\sim 7^{\circ}$ to $10^{\circ}$ Carrington longitudes (e.g., $\sim 12^{\circ}$ west of PSP). Further modeling of the PSP's magnetic connectivity is shown in Section 4.1.

At this time PSP was situated right between two active regions (ARs), AR 12737 and AR 12738, that could be the sources of the SEPs (see Figure 1(b)). Using extremeultraviolet (EUV) images from STA (EUVI) and SDO (AIA),
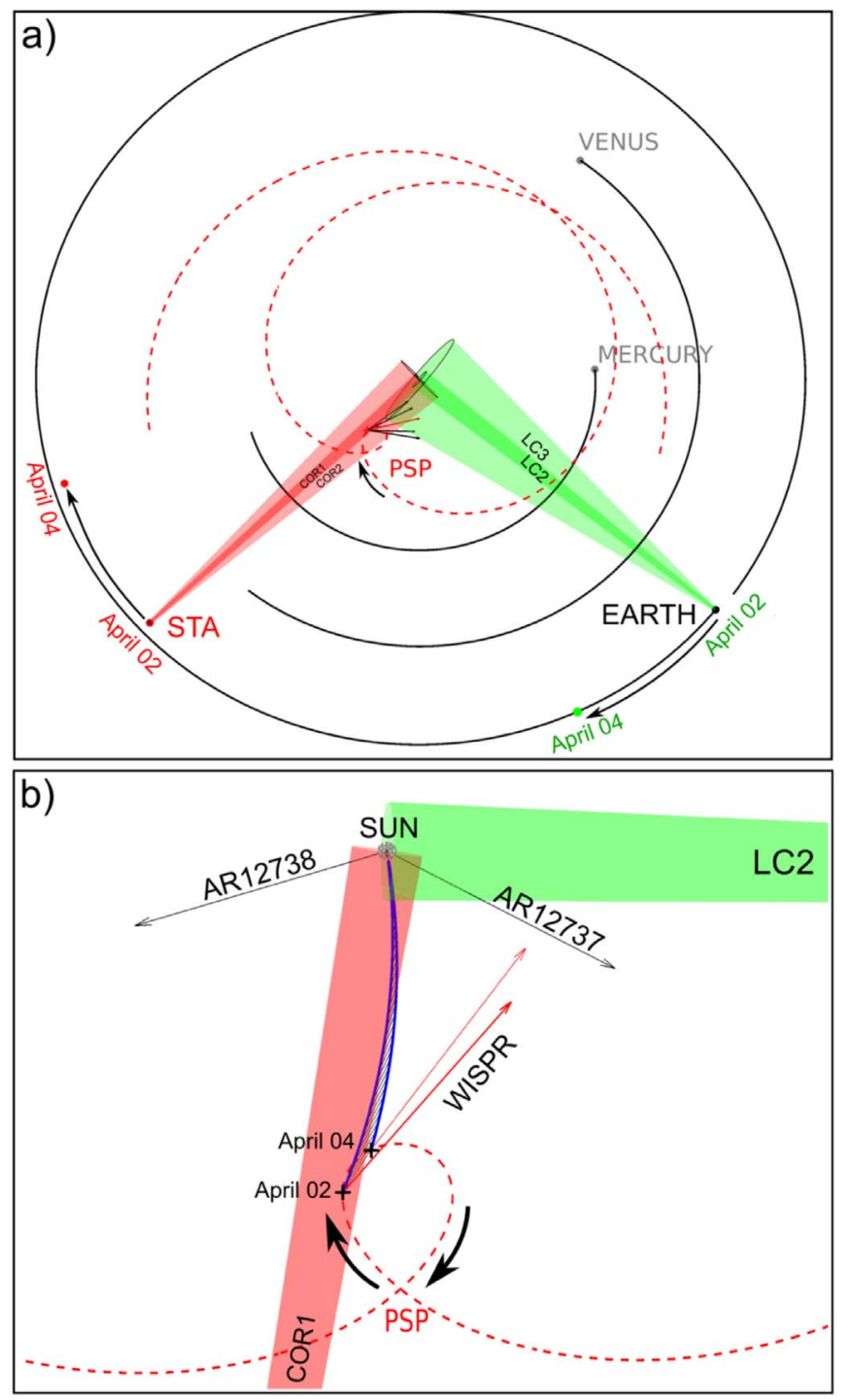

Figure 1. (a) A view of the ecliptic plane from ecliptic north showing the relative positions of the Sun and planets, STEREO-A, SOHO, and PSP in Carrington coordinates on 2019 April 2 and 4. The orbit of PSP is shown as a dashed red line. The fields of view of STA COR1 and COR2 and SOHO/ LASCO C2 and C3 are depicted with different shades of red and green, respectively. (b) A zoom-in version of panel (a) focusing closer to the second perihelion of PSP. The Parker spiral for a solar wind speed of $350 \mathrm{~km} \mathrm{~s}^{-1}$ connecting PSP to the solar surface is shown with blue lines. The central line of sight of the WISPR-I field of view is shown with red arrows originating from the PSP point position. The Sun is plotted to scale and the position of two active regions is shown with the upper two black arrows.

we determine the central position of the ARs in Carrington coordinates (CRLN and CRLT). AR 12737 was located at $\mathrm{CRLN}=62^{\circ}$ and $\mathrm{CRLT}=13^{\circ}$, that is westward from central meridian and above the solar equator, and AR 12738 was located at CRLN $=289^{\circ}$ and CRLT $=9^{\circ}$. STA was located at $\mathrm{CRLN}=352^{\circ} .188$ and Earth at CRLN $=89^{\circ} .611$ on April 2 00:00 UT. Hence, with respect to the Earth's viewpoint, AR 12737 was located in the eastern solar hemisphere and AR 12738 on the far sided, located behind the eastern solar limb; with respect to STA, AR 12737 was on the far side, behind the western limb, and AR 12738 was on the visible disk in the eastern hemisphere. Both ARs were of low magnetic complexity and their activity was relatively low but noticeable during the PSP encounter. 


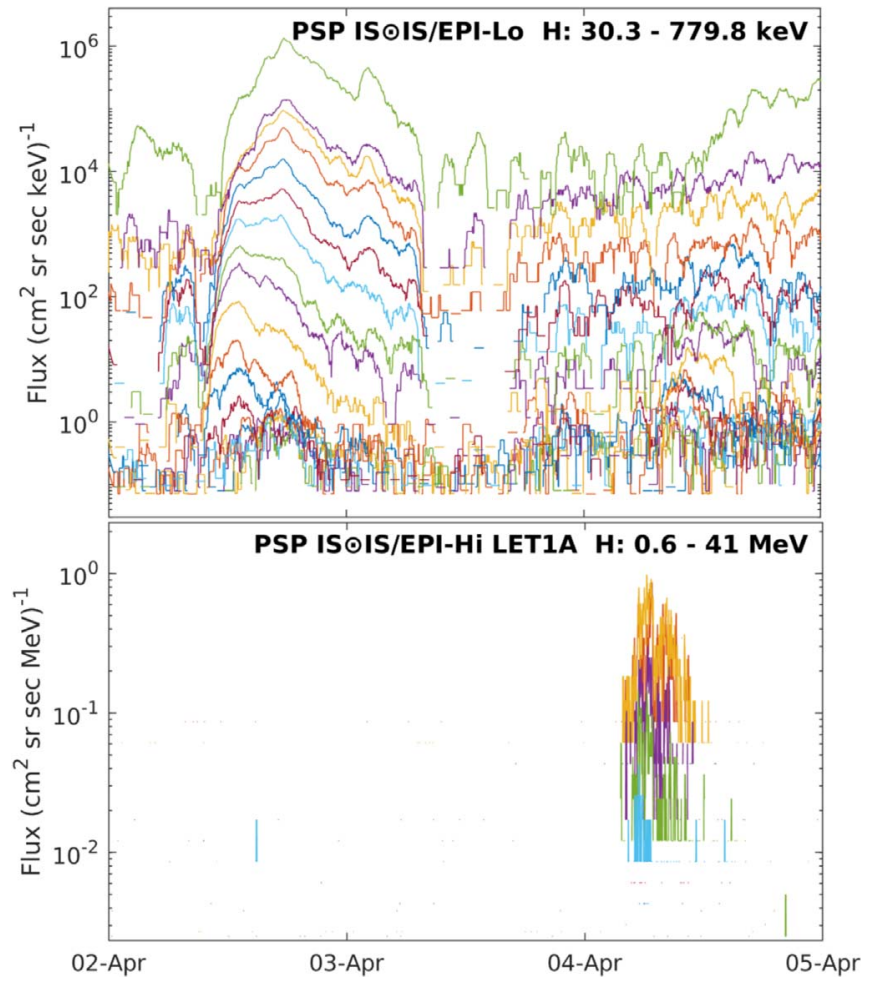

Figure 2. Time profiles of proton intensities from 2019 April 2 to 5. The top panel shows the measurements from IS $\odot$ IS/EPI-Lo at the indicated energy range, while the bottom panel shows the measurements from IS $\odot$ IS $/ \mathrm{EPI}-\mathrm{Hi} /$ LET1. The time profiles are produced using one-hour moving averages of data with one minute cadence.

\section{Observations}

\subsection{Overview of the SEP Events}

Even though solar activity was low, the Energetic Particle Instruments (EPI) of the Integrated Science Investigation of the Sun (IS $\odot$ IS: McComas et al. 2016) measured several SEPs during 2019 April 2 and 4 (see Leske et al. 2020; E. C. Roelof et al. 2020, in preparation) as PSP approached its perihelion. Two weak SEP events occurred two days apart (see Figure 2). The first event, on April 2, was detected by PSP at energies below several hundred $\mathrm{keV}$, without any increase in particles above $1 \mathrm{MeV}$; it was also relatively rich in heavy ions and the pitch-angle distributions was strongly anisotropic (see E. C. Roelof et al. 2020, in preparation). An estimate of the SEP release time is at 09:00 UT \pm 5 minutes. The second event, on April 4, was detected mostly at energies above $1 \mathrm{MeV}$. At lower energies, below $100 \mathrm{keV}$, the particle intensities were near the background levels. In contrast to the April 2 SEP event, that on April 4 was deficient in ions heavier than He. The onset time for this SEP event is found to be at around $03 \mathrm{UT}$.

\subsection{EUV Observations}

We begin with EUV observations of the low corona from STA and examine the activity of the two ARs and the region between them. Any eruptive activity within this region could potentially be linked to SEP measurements at PSP. AR 12737 would be more likely to be magnetically connected to PSP, being west of the probe, but it remained very quiet during the period of interest. Only one small-scale event occurs on April 2, 01:20 UT. It is located to the southwest of AR 12737 and is probably linked to the eruption of a CME observed later on
Table 1

Events on April 2 and 4 from EUV/WL Observations

\begin{tabular}{|c|c|c|c|c|}
\hline No. & $\begin{array}{l}\text { Time } \\
\text { (UT) }\end{array}$ & Event & $\begin{array}{c}\text { Wave Speed } \\
\left(\mathrm{km} \mathrm{s}^{-1}\right)\end{array}$ & $\begin{array}{c}\text { COR2 } \\
\text { (UT) }\end{array}$ \\
\hline \multicolumn{5}{|c|}{2019 Apr 2} \\
\hline 1 & $01: 45-02: 05$ & $\mathrm{~S}$ and $\mathrm{W}(?)$ & $270 \pm 85$ & 03:06 \\
\hline 2 & $02: 25-02: 55$ & $\mathrm{~S}$ and $\mathrm{W}$ & $155 \pm 60$ & $?$ \\
\hline 3 & $04: 45-05: 25$ & $\mathrm{~S}$ and $\mathrm{W}$ & $241 \pm 80$ & $06: 06$ \\
\hline 4 & $06: 50-07: 20$ & $\mathrm{~S}$ and $\mathrm{W}(?)$ & $\left(^{*}\right)$ & $?$ \\
\hline $5-6$ & $08: 50-09: 35$ & $\mathrm{~S}$ and $\mathrm{W} 1-2$ & $365 \pm 50$ & $10: 06$ \\
\hline 7 & $11: 15-11: 35$ & $\mathrm{~S}$ & $\ldots$ & $13: 06$ \\
\hline 8 & $13: 10-13: 55$ & $\mathrm{~S}$ and $\mathrm{W}$ & $172 \pm 100$ & $14: 36$ \\
\hline 9 & $15: 30-16: 05$ & $\mathrm{~S}$ and $\mathrm{W}$ & $415 \pm 80$ & $17: 06$ \\
\hline \multicolumn{5}{|c|}{2019 Apr 4} \\
\hline 10 & $03: 25-04: 25$ & $\mathrm{~S}$ & $\cdots$ & $(+)$ \\
\hline 11 & $05: 35-05: 55$ & $\mathrm{~S}$ & $\ldots$ & $(+)$ \\
\hline 12 & $11: 15-11: 35$ & $\mathrm{~S}$ & $\cdots$ & $(+)$ \\
\hline 13 & $15: 15-16: 18$ & $\mathrm{~S}$ and $\mathrm{W}$ & $380 \pm 80$ & $(+)$ \\
\hline 14 & $17: 55-18: 35$ & S & $\ldots$ & $(+)$ \\
\hline 15 & $22: 05-22: 30$ & $\mathrm{~S}$ & $\ldots$ & $(+)$ \\
\hline
\end{tabular}

Note. S: surge; W: EUV wave; ?: uncertain detection; $\left(^{*}\right)$ insufficient number of points to derive kinematics; $(+)$ signature possibly obscured by CME2.

April 2 by WISPR-I. The CME was also observed by STA/ COR2 at the western limb and also by LASCO/C3 at the eastern limb. A detailed description of this event is given later in this section.

On the other hand, we observe multiple ejections from AR 12738 throughout the PSP passage. In particular, over 20 narrow ejections, reminiscent of $\mathrm{H} \alpha$ surges, occurred between April 2 and 4. This AR was also the source of SEP events observed by IS $\odot$ IS near 1 au on April 20 and 21 (Schwadron et al. 2020; Wiedenbeck et al. 2020). In Table 1 we give an overall synopsis of the most important events in terms of material ejection and association to EUV waves. We also estimate the speed of the waves. All surges originated from the western edge of the leading sunspot and followed a western/ southwestern direction. Two of the $20+$ surges occurred in quick succession (events 5 and 6 in Table 1), were accompanied by EUV waves, and are connected to the April 2 SEP event.

The waves propagate in the southwestern direction (Figure 3) and typically last less than 30 minutes. Consequently, they cover a small distance (on average $\leqslant 0.8$ solar radii) on the visible disk before they become untraceable. The waves are visible over a narrow set of position angles. The 5 minutes cadence of EUVI images allows tracing of the fainter waves in only three to four frames, which leads to speeds with relatively high uncertainties (Table 1). Overall, we find propagation speeds consistent with past studies and of the order of the typical fast-mode speed for the quiet Sun (e.g., $\sim 300 \mathrm{~km} \mathrm{~s}^{-1}$ ). There are four events on April 2 and one on April 4 that have speeds exceeding the typical fast-mode speed.

In summary, we find two striking differences in the activity of AR 12738 between the two days (April 2 and 4). First, there is a much larger number of eruptive events associated with waves on April 2 (seven events) than on April 4 (one). The event of April 2 at 08:50-09:35 UT is the only one where two surges take place accompanied by two "fast" waves in quick succession. These differences may have a direct impact on the 


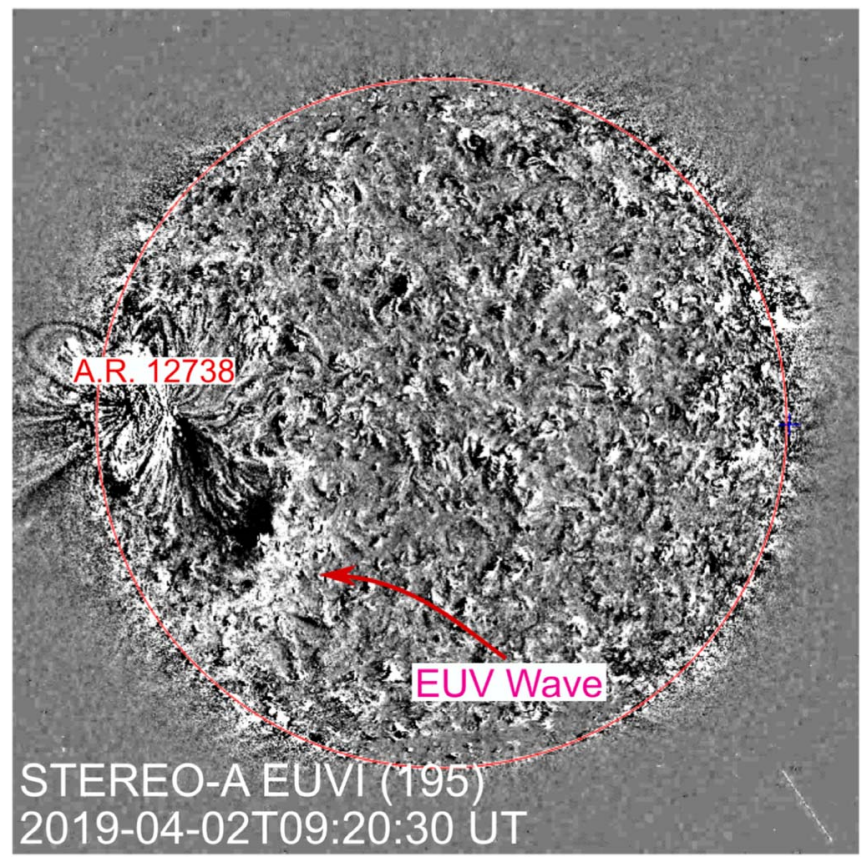

Figure 3. Running-difference image of EUVI-A 195 A images taken on 2019 April 2 at 09:20 UT. The front of an EUV wave at this time and AR 12738 that multiple ejections produced throughout the PSP passage are also marked.

characteristics of the SEPs measured in situ by PSP, as we will discuss later.

\subsection{White-light Observations}

We use white-light (WL) observations from STA/COR2, $\mathrm{SOHO} / \mathrm{LASCO}$, and PSP/WISPR to examine the eruptive activity in the high corona for the period April 2-4. We search for possible counterparts of the events in Table 1 and investigate whether other eruptions occurred higher in the corona without any clear signature in EUV images.

Based on the spacecraft locations and instrument FOVs (Figure 1), only eruptions from AR 12737 are likely to be observed by all three imagers. AR 12738 is located on the opposite side of the WISPR FOV and any eruptions along an eastern/southwestern trajectory (as suggested by the EUV surges) would be halos and may be undetectable by COR2, depending on their size.

The time interval of interest here (April 2-4) coincided with the "deep-field" SECCHI/COR2-LASCO/C3 campaign, which consists of high cadence (5 minutes for COR2, 7 minutes for $\mathrm{C} 3$ ) and long exposures for COR2 (36 s, C3 remained at the $17 \mathrm{~s}$ synoptic exposure). Because of on-board processing constraints, no C2 image was acquired between April 1 and 4.

The purpose of the "deep-field" campaigns is the investigation of small-scale solar wind activity. In our case, the longer exposures are useful to search for eruptive activity in relation to the SEP events, which is expected to be faint, as discussed above. Our extensive examination of the COR2 and C3 time series between April 2 and 4 resulted in the following findings.

April 2: A CME emerges over the western limb, relative to STA, at around 03:06 UT (Figure 5(a)). The same event is detected by C3 over the eastern limb at 4:40 UT. The source region is located on the Earth-facing part of the solar disk, the event shows no signatures of a shock or pressure wave, and it

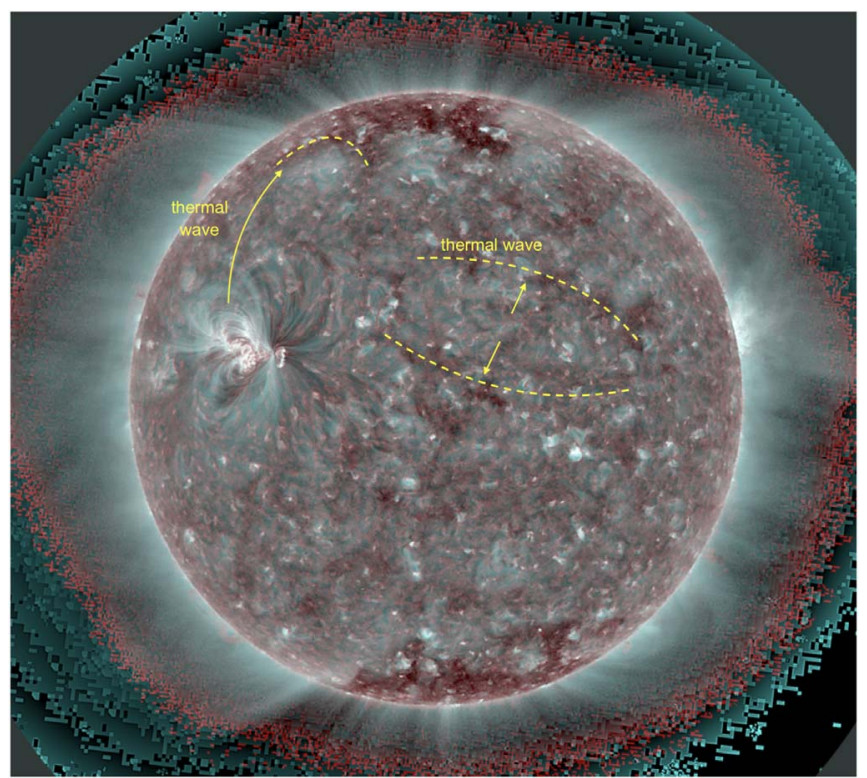

Figure 4. Composite of EUVI-A $195 \AA$ (silver) and $304 \AA$ (red) waveletprocessed images taken on 2019 April 3 at 20 UT. The fronts of the two thermal waves at this time and their expansion directions as seen in the accompanying movie are also marked. The thermal waves are the low atmosphere counterparts of the two components of CME2, as discussed in the text. An animation of this figure is available in the online Journal. The animation, which is not annotated, runs from 22:00 UT on 2019 April 1 to 01:00 UT on April 5.

(An animation of this figure is available.)

moves with a constant speed through the COR2 FOV. The event is in process when IS $\odot$ IS detects the high- $Z$ particles but we can find nothing in the CME behavior unusual around that time. The SEP injection timing, and the CME location and kinematics, argue against this CME being the source of the particles. As we examine and discuss further later, the source(s) are likely associated with the EUV surges.

Regarding the surges, we detect several wave-type ejections (Vourlidas et al. 2017) entering the COR2 FOV at approximately 6:06 UT, 10:06 UT, 12:06 UT, 14:36 UT, and 17:06 UT, most likely corresponding to surges \#3, \#5-6, \#7, \#8, \#9 (Figures 5(a), (b)). The signatures remain visible for about $2 \mathrm{hr}$ in each event. We note that an eruption over the eastern limb is detected in EUVI-A starting at approximately 11:20 UT, which hinders a robust association of any whilelight signatures over the east limb after that time, hence the question mark for \#7 in Figure 5(b). In addition, all while-light signatures are very faint and would generally escape attention for the specific search for the counterparts of the EUV surges. Given the southwestern evolution of the EUV surges, we expect the bulk of the CME (if it exists) to lie behind the COR2 occulter and thus be undetectable. But these transients would lie close to the C3 sky plane and may be detectable. Based on the comparison between the COR2 and C3 high-cadence movies (not shown here), we believe that the front seen at 13:06 UT (Figure 5(b1)) is the eastern extension of \#5-6, and possibly \#7.

April 3: A CME heading to the northeast enters the COR2 FOV at 22:06 UT on April 2 and evolves through April 4, when it leaves the COR2 FOV at $\sim 20$ UT (Figures 5(c)-(e)). EUV loop structures connected to AR 12738 are seen lifting off, followed by a faint "thermal wave" (Robbrecht \& Wang 2010) spreading northward from the AR (Figure 4) 

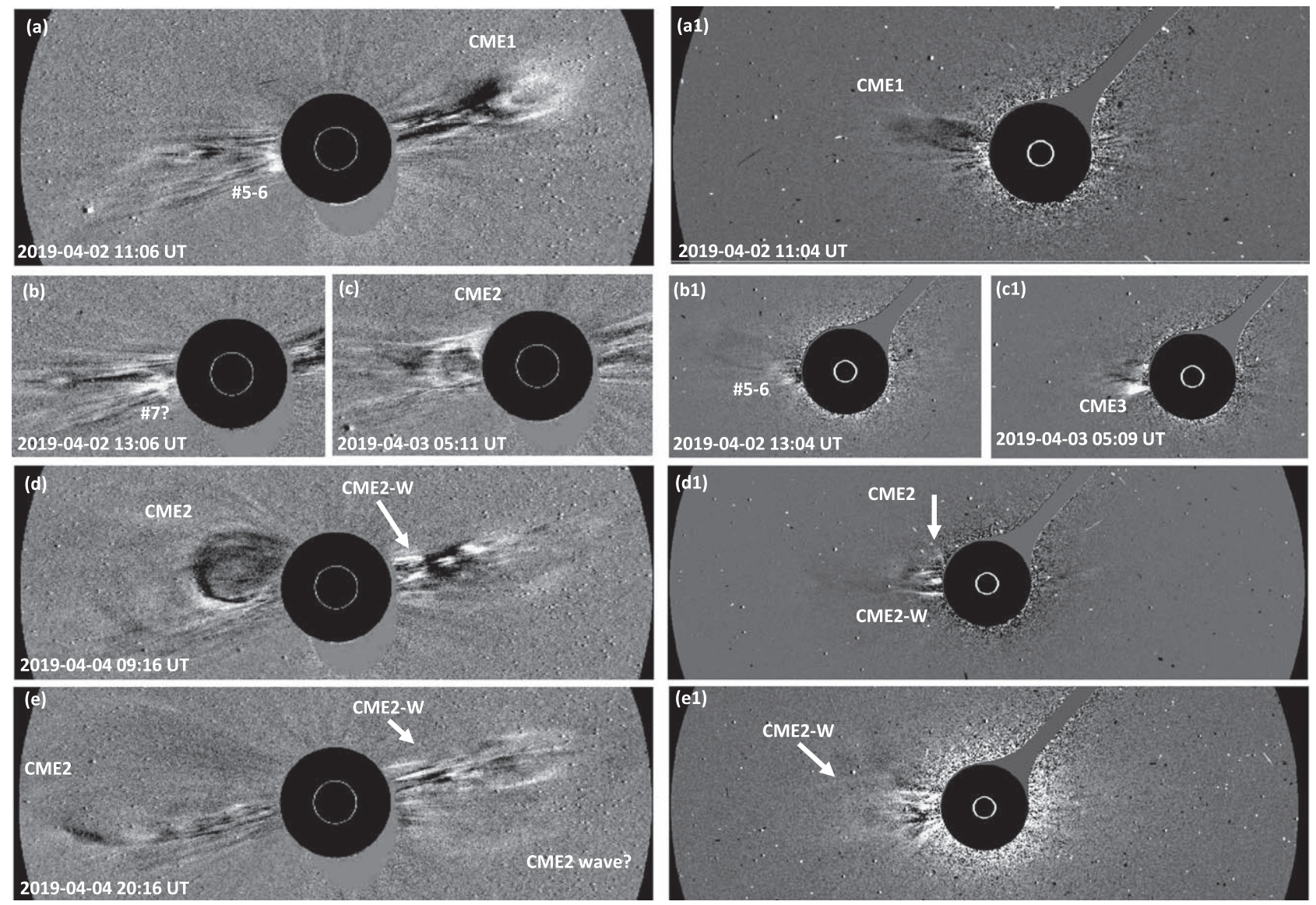

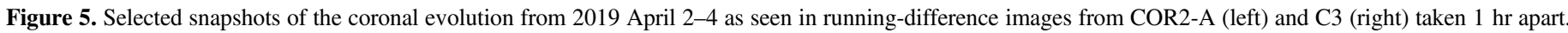

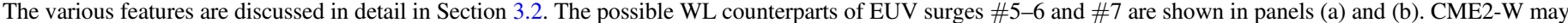

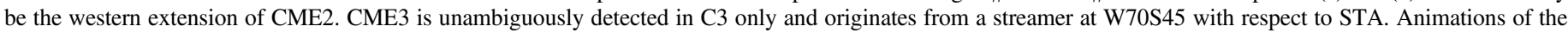

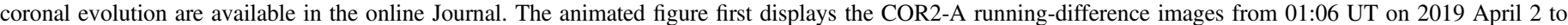
23:16 on 2019 April 4 then shows the C3 running-difference images from 00:56 UT on 2019 April 2 to 23:21 on 2019 April 4.

(An animation of this figure is available.)

from 03 UT on April 3 to 08 UT on April 4. In addition, another "thermal wave" appears to the east of AR 12739 at approximately 08 UT on April 3 and spreads parallel to the equator until 14 UT on April 4. The signatures of these waves are very faint, seen in EUVI-A $195 \AA$ and $171 \AA$ waveletprocessed movies only when played with high frame rates. These signatures, however, are quite common during solar minimum and constitute the response of the lower atmosphere to a streamer-blowout CME (SBO-CME). In this case, the northeastern part of the CME is seen clearly in COR2 (CME2 in Figures 5(c)-(e)) but the east-west extension, being out of the sky plane and very slow, is not detected as a propagating front. Instead, it can be seen in the hour-long runningdifference movies (in both COR2 and C3) as "opening jaws" (CME2-W in Figures 5(d) and (e)). This event, which again could escape detection easily, is likely to lead to a reconfiguration of the global magnetic field and as such has important implications for the SEP event detected on April 4. LASCO/C3 detects another CME ("CME3"), starting late on April 2, along the southeastern streamer. The EUVI-A $195 \AA$ observations show weak thermal signatures around a quiet-Sun area at approximately S45W70, relative to the STA subsolar point. The CME is detected clearly in C3 (Figure 5(c1)) and it has exited the $\mathrm{C} 3 \mathrm{FOV}$ by the time of CME2.
April 4: The SBO-CME continues throughout the day (Figures 5(d) and (e)). Eventually we are able to detect its expanding outline, from 11:16 UT onward, as a very faint front expanding to the southeast (labeled "CME2 wave?" in Figure 5(e)). The CME appears directed toward the PSP location and we posit that the faint particle event detected on April 4 by EPI-Hi (Leske et al. 2020) is associated with this event.

\section{Modeling \\ 4.1. Magnetic Connectivity of PSP}

We start the modeling with the estimation of PSP's magnetic connection to the solar surface. We use magnetic field properties of the background corona modeled via the magnetohydrodynamic around a sphere thermodynamic (MAST) model $^{4}$ (see Lionello et al. 2009; Riley et al. 2011). An important aspect is that AR 12738 is invisible to Earth until April 8, so the magnetic maps of Carrington rotation (CR) 2215 are prone to a large uncertainty in the regions of interest. For this analysis we use MAST cubes for both CR 2215 and CR 2216 to address the differences in the PSP's connectivity between the two models. To model the eruptions

4 http://www.predsci.com/ 

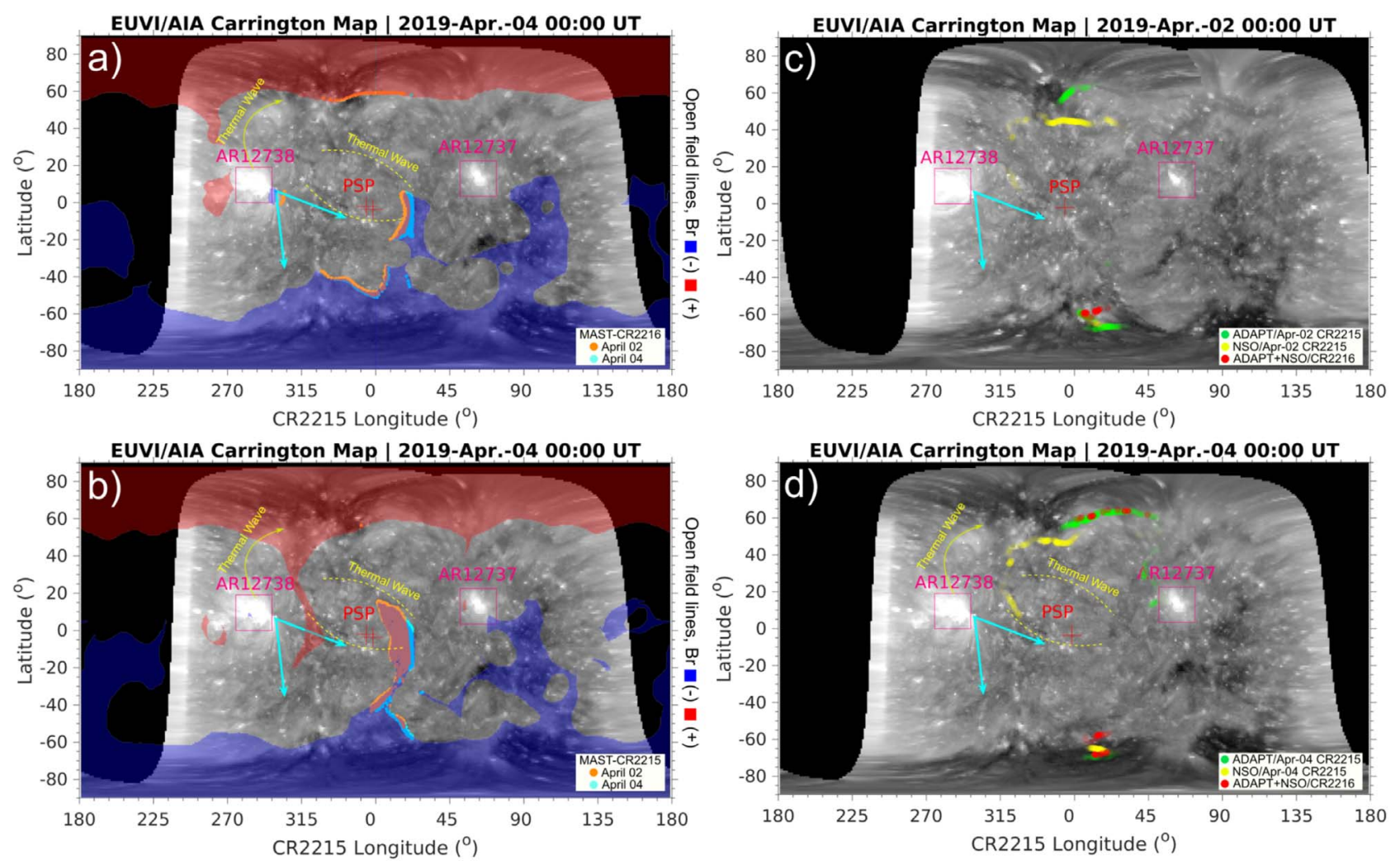

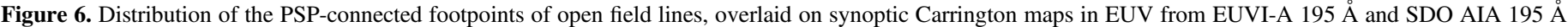

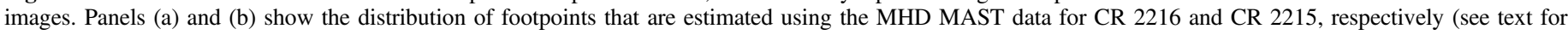

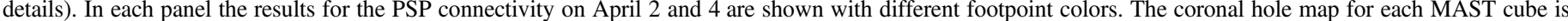

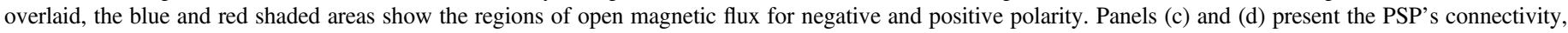

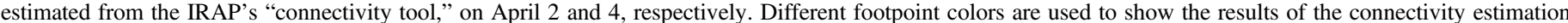

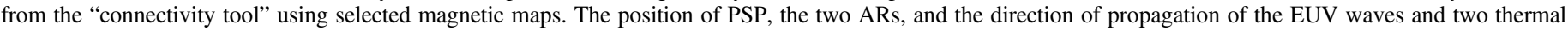
waves observed on April 3 are marked in each panel.

in Section 4.2 we use the MAST model of CR 2216. For CR 2216, when AR 12738 has been updated in the magnetic map, the field polarity is in agreement with the PSP measurements. For CR 2215 there is an inconsistency in the position of the heliospheric current sheet and therefore the magnetic field polarity that PSP should measure during the encounter.

We consider the open magnetic field lines and trace these lines from the solar surface to the boundary at $30 R_{\odot}$. To determine which of the open field lines traced from MAST data (MAST-FLT) connect to PSP, we consider a region around the connection of a nominal Parker spiral of $350 \mathrm{~km} \mathrm{~s}^{-1}$ (average speed using PSP/SWEAP measurements) at $30 R_{\odot}$. We register every field line located inside angular distances ranging from $5^{\circ}$ to $15^{\circ}$. Since the interplanetary magnetic field measured by PSP is highly structured (e.g., Bale et al. 2019; Rouillard et al. 2020), we expect that the magnetic field lines could meander in space at a characteristic angular scale of about $10^{\circ}$, which is similar to that associated with the size of solar supergranules (Giacalone \& Jokipii 2004).

In Figures 6(a) and (b) we show the results of the PSP's magnetic connectivity from MAST-FLT of CR 2216 (panel (a)) and CR 2215 (panel (b)) using an extent of $10^{\circ}$. We observe some differences in the calculated connectivities between the two CR MAST cubes; they are due to the different input photospheric magnetograms. We overplot in
Figures 6(a) and (b) the coronal hole maps provided by Predictive Sciences Inc. (PSI ${ }^{5}$ ) for the two MHD cubes. The presence of mid-latitude and equatorial coronal holes has an important impact on the determined connectivity. For CR 2215 we find that PSP is mainly connected to the equatorial coronal hole on April 2 and 4 (Figure 6(b)) and there is an extension of the connectivity to the southern coronal hole boundary on April 4. For CR 2216, PSP is connected to the boundaries of the equatorial and southern/northern coronal holes on April 2. Progressively the connectivity changes toward the core of the equatorial coronal hole on April 4 (Figure 6(d)), and there is also a connection to the southern coronal hole boundary. This connectivity is broadly consistent with the PSP/SWEAP measurements throughout the period April 2-4, as we discuss next.

PSP/SWEAP has measured two regimes from April 1 to 5, starting with a period of dense and slow solar wind $\left(\sim 300 \mathrm{~km} \mathrm{~s}^{-1}\right)$ followed by a period of significantly more tenuous and slightly faster solar wind $\left(>400 \mathrm{~km} \mathrm{~s}^{-1}\right)$ near perihelion. On April 3 PSP was super-corotating and appears to have crossed a magnetic separatrix region (see E. C. Roelof et al. 2020, in preparation). Around this crossing there is an abrupt drop in the April 2 SEP intensities and there are significant changes in the solar wind properties. From Figures 3 and 4 of Rouillard et al. (2020) we see that PSP remained near the southern edge of a streamer throughout its second

5 http://www.predsci.com/hmi/coronal_hole_map.php 
encounter, and from April 2 to 5 it exited streamer flows to enter significantly less dense wind. On April 2 PSP was still connected to the streamer flows, where high densities were measured. The connectivity from MAST (CR 2216) is located on the periphery of the equatorial coronal hole, sampling field lines that are indeed likely channel streamer flows. After April 3, PSP exits the streamer, the plasma density drops suddenly, and MAST suggests that magnetic connection changes progressively to the core of the equatorial coronal hole.

To give a further context to the estimation of PSP's connectivity we also used the IRAP's "connectivity tool" accessible at http://connect-tool.irap.omp.eu/. This tool performs simultaneous estimates of connectivity based on different combinations of models and boundary conditions rather than relying on a single model/data set. As done for MAST above, it assumes a Parker spiral to estimate magnetic connectivity to the outer boundary of the the coronal model it considers. Here we use the potential field source surface (PFSS) model of different synoptic magnetic maps. For PSP the tool provides two solutions, one for slow $\left(300 \mathrm{~km} \mathrm{~s}^{-1}\right)$ and one for fast $\left(800 \mathrm{~km} \mathrm{~s}^{-1}\right)$ solar wind. Since PSP appears to have measured slow solar wind $\left(\sim 300-350 \mathrm{~km} \mathrm{~s}^{-1}\right)$ near the perihelion we use only the "slow solutions" of the tool. We consider solutions based on Carrington maps of the solar surface magnetic field from the Air Force Data Assimilative Photospheric Flux Transport model (ADAPT) and the National Solar Observatory (NSO) for CR 2215 and CR 2216.

In Figures 6(c) and (d) we show the results of the PSP's magnetic connectivity from the "connectivity tool." The zones of high probability of connectivity for PSP are overplotted on the composite EUV Carrington maps constructed from SDO AIA and STEREO EUVI observations. From April 2 to 4 the connectivity does not change significantly but there are some noticeable differences. On April 2, regions with the highest probability of connectivity are located mostly near the northern and southern coronal hole boundaries (Figure 6(c)). Two days later, on April 4, those regions remain near the northern coronal hole boundary but there are also some high-latitude wellconnected regions inside the southern coronal hole (Figure 6(d)). The connectivity from NSO on April 4 shows that there are a few connected regions with lower probability of connectivity, closer to the ecliptic plane near the boundary of a small equatorial coronal hole, while the connectivity from ADAPT shows an additional connection near AR 12737.

Overall, the results of this analysis suggest that PSP is connected to a southern coronal hole and an equatorial one and near their boundaries. There are some differences in the PSP connectivity from the models presented, which are mainly due to the different input photospheric magnetograms. The 3D MHD model MAST gives magnetic footpoints rooted in a lowlatitude coronal hole, in contrast to PFSS, which suggests magnetic connection to the polar regions. AR 12737 was nearequatorial, so although the PFSS favors the connection to the southern polar coronal hole (at least for the CR 2216 solutions), this type of mapping to the northern polar coronal hole should not be ruled out. However, we note that PSP throughout the second encounter measured mostly negativepolarity wind from PSP/FIELDS measurements, pointing mostly to a connection at a region of negative polarity (i.e., southern/equatorial coronal holes).
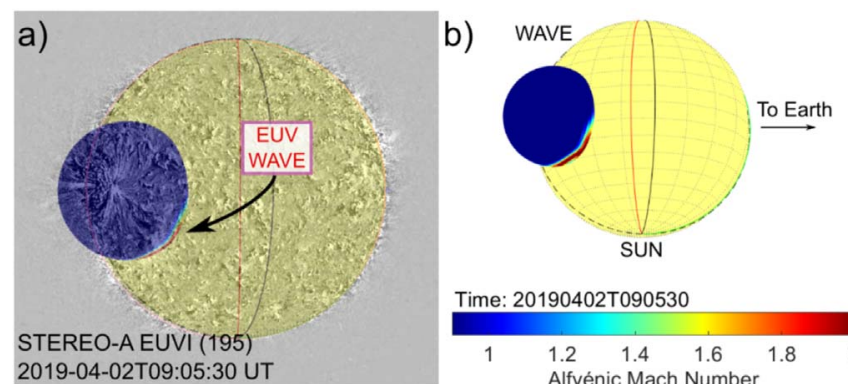

2019-04-02T09:05:30 UT

c)

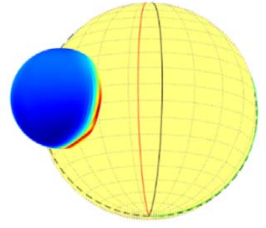

d)
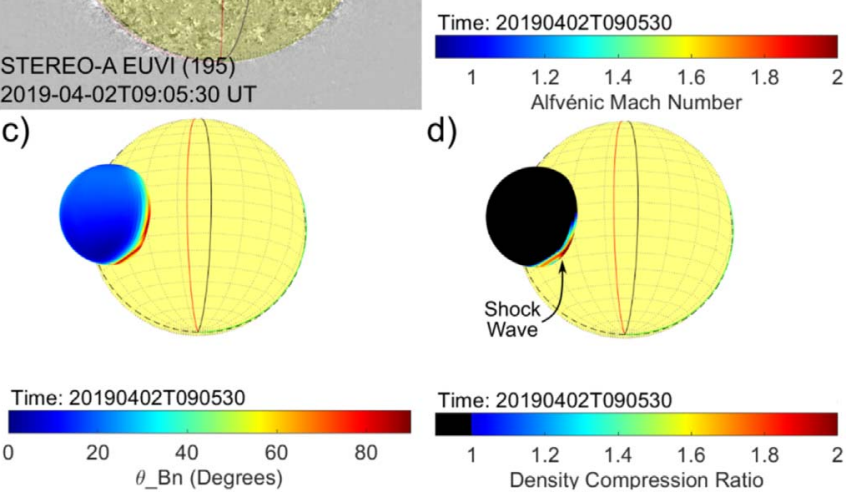

Time: 20190402T090530

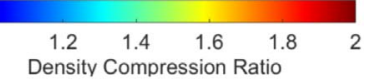

Figure 7. Results of the 3D properties along the surface of the reconstructed pressure wave front for the event \#5-6 at 09:05 UT. Panels show (a) an image of EUVI-A at 09:05:30 UT with the reconstructed wave colored by the 3D distribution of the Alfvénic Mach number, and 3D distributions of (b) the Alfvénic Mach number, (c) the magnetic field obliquity with respect to the shock normal, and (d) the density compression ratio.

\subsection{D Modeling of the April 2 EUV Waves}

As discussed earlier, event \#5-6 (Table 1) is the most likely source for the high- $Z$ SEP event on April 2, based on the eruption times. Here, we test this assertion by reconstructing and modeling the EUV wave to estimate the shock parameters and magnetic connectivity to PSP using the methods presented in previous papers (Kouloumvakos et al. 2019, and references therein).

We start with the 3D reconstruction of the frontal structure of the EUV wave. We fit an ellipsoid model to the EUV wave's outermost part using observations mainly from a single viewpoint (STA EUVI) since the wave is almost invisible from SDO AIA. We assume self-similar expansion from the source region and consider the full 3D ellipsoids although the EUV wave signature is localized to a narrow $\left(\sim 30^{\circ}\right)$ area on the surface. This approach allows us to get the global properties of the wave. From the time sequence of the fitted ellipsoids we derive the 3D expansion speed of the wave. Then we compute the shock parameters at the wave front using the plasma and magnetic field properties of the background corona modeled from MAST. We consider the shock Alfvénic Mach number $\left(M_{\mathrm{A}}\right)$ in this study, e.g.,

$$
M_{\mathrm{A}}=V_{\mathrm{sh}}^{\prime} / V_{\mathrm{A}}=\left(V_{\mathrm{sh}}-\boldsymbol{V}_{\mathrm{sw}} \cdot \hat{\boldsymbol{n}}\right) / V_{\mathrm{A}}
$$

where $V_{\mathrm{sh}}, V_{\mathrm{sw}}$, and $V_{\mathrm{A}}$, are the shock speed, the solar wind speed, and the Alfvén speed along the shock normal direction, respectively. In addition, we compute the magnetic field obliquity with respect to the shock normal $\left(\theta_{\mathrm{Bn}}\right)$ and the density compression ratio $(X)$ (see Kouloumvakos et al. 2019).

In Figure 7 we give a 3D view of the modeled wave and overlay the different shock parameters that resulted from the modeling. Panel (a) shows an image of EUVI-A at 09:05:30 UT with the reconstructed wave colored by the 3D distribution of the Alfvénic Mach number. The arrow points to a location where the model predicts the formation of a shock 
$\left(M_{\mathrm{A}}\right.$ and $\left.X>1\right)$. This coincides with the location of the bright EUV front, strongly suggesting that the EUV wave could be the source of the SEPs. Panel (b) shows the Alfvénic Mach number without the EUV image for clarity and the viewpoint is adjusted to CRNL $=353^{\circ}$ and CRLT $=-15^{\circ}$. Panels (b)-(d) show the $3 \mathrm{D}$ distribution of $M_{\mathrm{A}}, \theta_{\mathrm{Bn}}$, and $X$ along the ellipsoid surface. The modeling shows that a shock has formed along a narrow region at the flanks of the EUV wave. In this region, we find that $M_{\mathrm{A}}$ values range from $\geqslant 1$ to $\sim 3$.

The magnetic geometry $\left(\theta_{\mathrm{Bn}}\right)$ is generally quasi-parallel but in the regions where a shock has formed $\theta_{\mathrm{Bn}}$ is quasiperpendicular (panel (c)), changing slightly to oblique near the end of our modeling. A quasi-perpendicular geometry near the flanks of the modeled wave is expected since the expansion is almost perpendicular to the magnetic field lines. Panel (d) shows that most of the pressure wave has $X<1$ (black region), which results from a combination of low $M_{\mathrm{A}}$ values $(\ll 1)$ and the magnetic geometry. Most of pressure wave is unlikely to have developed into a shock. Again, close to the propagation direction of the EUV wave the $M_{\mathrm{A}}$ values of $\sim 2$ are associated with $X \sim 1.5$ from 09:05 UT to 09:10 UT. Our 3D modeling therefore suggests that the EUV wave has steepened into a shock that is almost supercritical $\left(M_{\mathrm{A}}>M_{\mathrm{c}}\right.$ with $\left.M_{\mathrm{c}} \sim 2\right)$ in certain regions and hence may be capable of accelerating particles.

The next step to consider is the magnetic connectivity of the modeled shock with PSP, including the evolution of the shock parameters for the magnetic field lines that thread the wave surface and connect to PSP. For this analysis we use the wellconnected field lines calculated from PFSS (i.e., IRAP Magnetic Connectivity Tool) and those derived from the MAST 3D cubes. We perform a temporal analysis of the shock properties for these field lines. We infer the shock parameters at the cobpoint for each field line and calculate their average values at every time step. It is worth noting that among the PFSS models we find a prompt magnetic connection of the shock wave to PSP only for the NSO magnetogram of CR 2215. Other models show either an extremely late connection or no connection at all. For the reasons explained in Section 4.1 the magnetic maps are prone to a significant uncertainty in the photospheric regions that are of interest here.

Figure 8 shows the evolution of the average $M_{\mathrm{A}}$ (panel (a)) and $X$ (panel (b)) at the well-connected field lines. We have time-shifted the measurements by 6.76 minutes to be comparable with the PSP in situ measurements. ${ }^{6}$ We estimate that the first magnetic connection of PSP to the shock is established at around 08:59 UT (PFSS) to 09:06 UT (MAST-FLT $\left(15^{\circ}\right)$ ). The evolution of the shock parameters does not differ significantly among the different connectivity models and both parameters are nearly constant during the modeling interval. We find that $\left\langle M_{\mathrm{A}}\right\rangle=1.99 \pm 1.02$ and $\langle X\rangle=1.52 \pm 0.20$ (excluding a clear outlier at $\sim 09$ UT).

According to E. C. Roelof et al. (2020, in preparation) the spectral index for the ${ }^{4} \mathrm{He}$ omnidirectional intensity was approximately -4.5 throughout the event ( -11 on momentum). From diffusive shock acceleration theory the shock acceleration leads to a power-law spectrum that depends on the density compression ratio with an index on momentum of $-3 X /(X-1)$. From the mean compression ratio $\langle X\rangle=1.52 \pm 0.20$ of the well-connected shock regions to

\footnotetext{
6 We account for the light travel time between the PSP and 1 au. The time reference frame of the shock model is at $1 \mathrm{au}$.
}
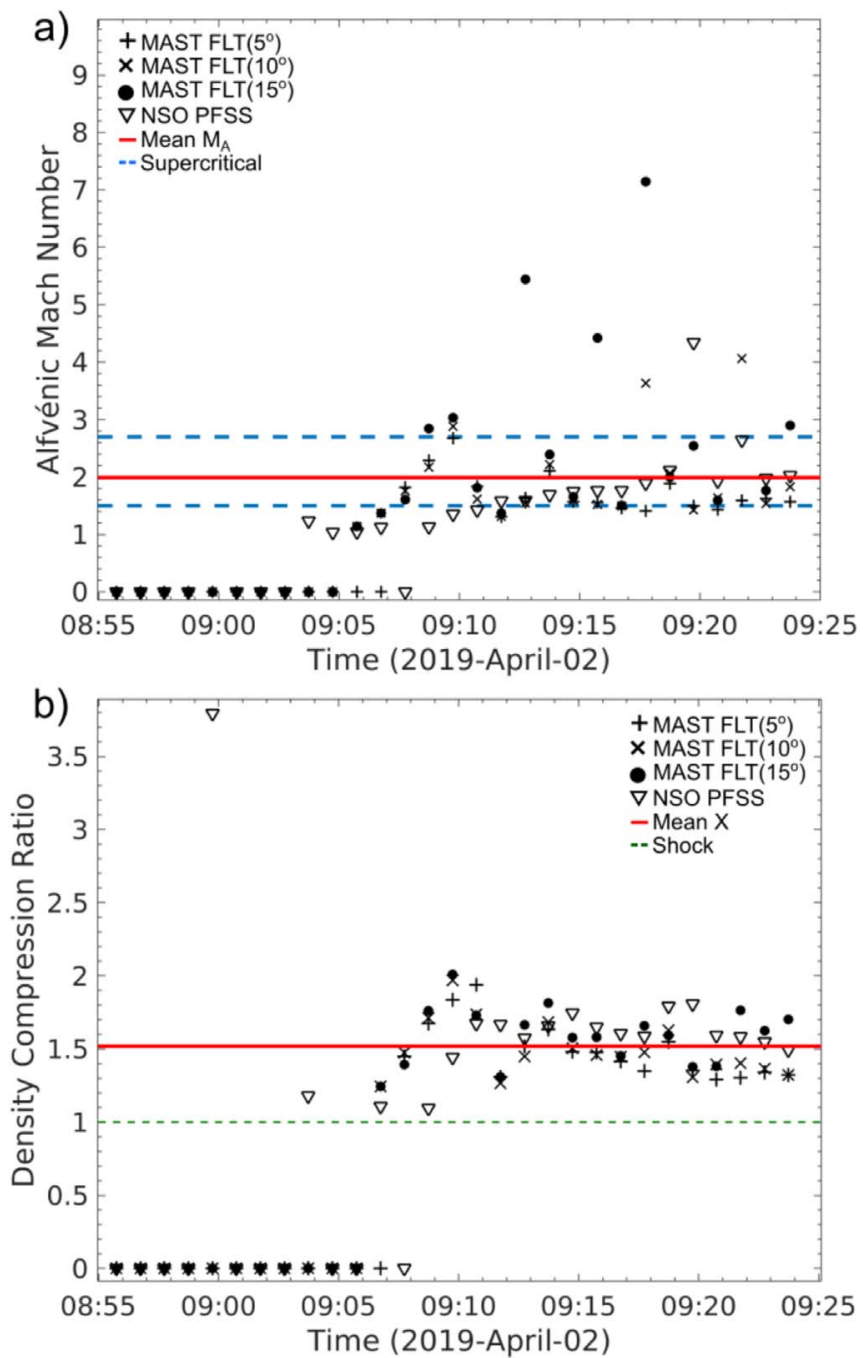

Figure 8. Evolution of the mean values of two shock parameters for PSPconnected field lines, from 08:55 UT to 09:25 UT. (a) Mean Alfvénic Mach number; (b) mean density compression ratio.

PSP we find a spectral index of -3.4 on energy $(-8.8$ on momentum). Considering that $X$ deviates from the mean value by $\sim 0.2$ during the modeling interval, this gives high-low limits for the spectral index of $[-2.6,-5.2]$ on energy $([-7.2$, $-12.4]$ on momentum).

\subsection{Shock Formation at Other Eruptions}

The modeling of event \#5-6 shows that the merged EUV wave was fast enough to form a quasi-perpendicular shock that could explain the SEP event measured at PSP on April 2. However, there were other EUV waves with similar speeds (e.g., \#9 and \#13 in Table 1), which are not associated with any SEP detection at PSP. Is this a matter of connectivity or shock formation?

Instead of modeling individually each of the EUV waves of Table 1, we construct a basic model, using the products and results of the 3D modeling of Section 4.2. From this model our aim is to establish a threshold for shock formation at the field lines connected to PSP. From the definition of the Alfvénic Mach number (see Equation (1)), we have that the minimum condition to have a shock wave solution in the RankineHugoniot $(\mathrm{RH})$ formalism is $M_{\mathrm{A}} \geqslant 1$, so $V_{\mathrm{sh}}^{\prime} \geqslant V_{\mathrm{A}}$. From this 


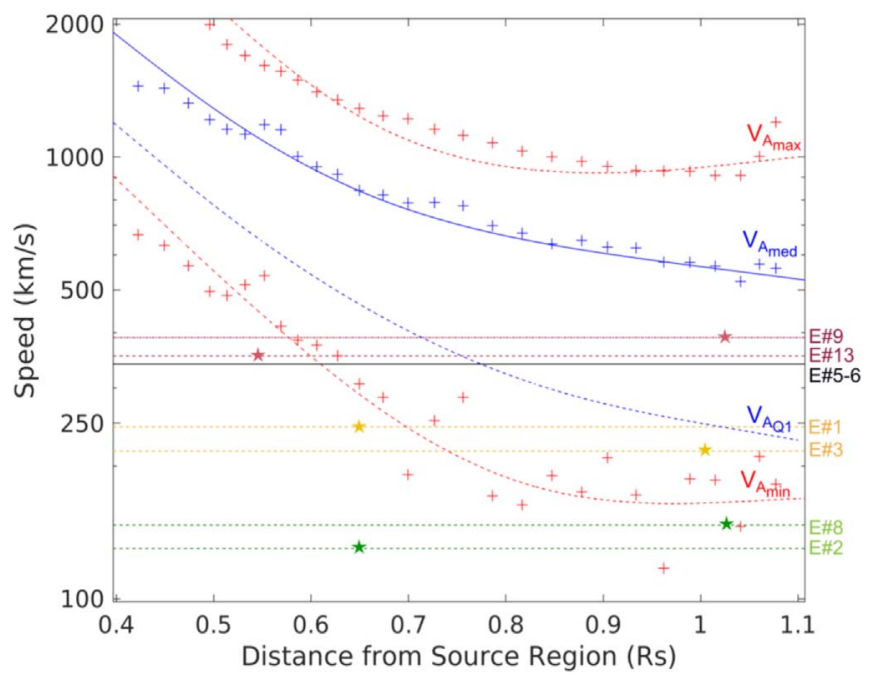

Figure 9. Profiles of the Alfvén speed distribution parameters at the PSPconnected field lines that wave \#5-6 intersected during its expansion, as a function of distance from the source region (AR 12738). The colored horizontal lines show $V_{\mathrm{sh}}^{\prime}$ for each of the EUV waves of Table 1. The remaining lines show the minimum/maximum (red dashed lines), first quartile (blue dashed line), and median (blue line) $V_{\mathrm{A}}$ values (see text for details).

condition and the wave speeds in Table 1 we can examine whether shock formation is possible during the eruption.

To construct the model, we consider the PSP well-connected field lines that wave \#5-6 intersected during its expansion. For each field line we calculate $V_{\mathrm{A}}$ at the intersection points with the wave surface, and the distance between the intersection points and the wave source region. This calculation provides $V_{\mathrm{A}}$ values along the shock wave's path. We compute different statistical values of $V_{\mathrm{A}}$ (first/last decile-10th/90th percentile, first/third quartile-25th/75th percentile, median or second quartile-50th percentile) in discrete bins of the distance. For the extreme minimum/maximum values of the $V_{\mathrm{A}}$ distribution, e.g., $V_{\mathrm{A}_{\min }}$ and $V_{\mathrm{A}_{\max }}$ respectively, we have used the first/last decile values to avoid any effect from outliers on our statistics. The final result is presented in Figure 9, where we show the minimum/maximum limits (red dashed lines), first quartile (blue dashed line), and median (blue line) $V_{\mathrm{A}}$ values as a function of distance from the source region. At a distance of $1 R_{\odot}$ from the source region, the lower limit of $V_{\mathrm{A}}$ is around $165 \mathrm{~km} \mathrm{~s}^{-1}$, the first quartile value is $250 \mathrm{~km} \mathrm{~s}^{-1}$, and the median $V_{\mathrm{A}}$ is $565 \mathrm{~km} \mathrm{~s}^{-1}$. Away from an active region a typical $V_{\mathrm{A}}$, at the same distance, is around $500 \mathrm{~km} \mathrm{~s}^{-1}$ (e.g., see Figure 4 of Mann et al. 2003). A shock solution is possible at the PSPconnected field lines if a wave passing through this region has much higher speed than the minimum $V_{\mathrm{A}}$. We estimate that $\boldsymbol{V}_{\mathrm{sw}} \cdot \hat{\boldsymbol{n}} \sim 25 \mathrm{~km} \mathrm{~s}^{-1}$ and we use this value to calculate $V_{\mathrm{sh}}^{\prime}$ for each event (see Equation (1)).

In Figure 9 we plot $V_{\text {sh }}^{\prime}$ using the average speed of the EUV waves of Table 1 (colored lines). For event \#5-6 $V_{\mathrm{sh}}^{\prime}$ is higher than the lower limit of $V_{\mathrm{A}}\left(V_{\mathrm{Amin}}\right)$, so $M_{\mathrm{A}}$ is greater than unity. Shock formation is possible for this event, consistent with the results presented earlier. For the other events we find that events \#9 and \#13 produce EUV waves with an average $V_{\text {sh }}^{\prime}$ much higher than the minimum $V_{\mathrm{A}}$ threshold, the speed is close to the threshold for events \#1 and \#3, and it is well below it for events \#2 and \#8. Therefore a weak shock may have formed for events \#1 and \#3, while shock formation is not possible for events \#2 and \#8.

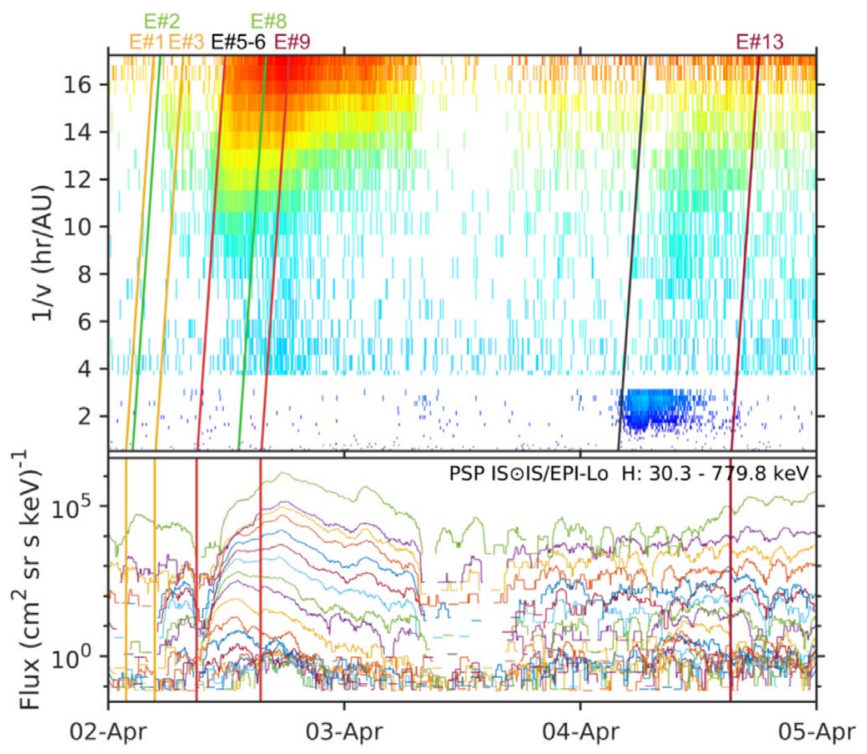

Figure 10. Top panel: the inverse particle velocity is plotted vs. time for EPILo/Hi, from April 2 to 5. Bottom panel: time profiles for EPI-Lo H rates. The colored lines indicate the expected locations of particles assuming an injection at the onset time of the EUV waves and a path length of 0.168 au (e.g., solar wind speed $350 \mathrm{~km} \mathrm{~s}^{-1}$ ).

Another aspect we examine here is that for some events the wave propagates a short distance and it seems to damp very quickly. In Figure 9 we overplot for each event a colored star that shows the maximum distance for which the wave could be traced. For example, for events $\# 13$ and $\# 1$ the waves degenerate before they reach the region where $V_{\mathrm{A}}$ becomes minimum. In this case either a weak shock wave may have formed but it quickly degenerated before a connection to PSP was established or a shock simply failed to form. This analysis suggests that shock formation and magnetic connection to PSP is possible for events \#5-6 and \#9 on April 2.

\subsection{Connecting the Modeling with SEP Observations}

The modeling of the eruptions suggests that two of the events could produce shock waves that were magnetically connected to PSP during their expansion. For those events we search for a connection with the SEP measurements. We suppose that waves that were not associated with shock formation could not lead to separate SEP injections.

In Figure 10 we show particle measurements from PSP/ IS $\odot$ IS. The inverse particle velocity is plotted versus time for EPI-Lo/Hi in the top panel and the time profiles for EPI-Lo $\mathrm{H}$ rates are plotted in the bottom panel. If we assume that the particle injection profile is a step function and that particles have traveled to PSP without significant scattering, then we should observe clear velocity dispersion. The arrival time at PSP of the first particles as a function of the inverse particle velocity would appear as a straight line boundary. This would be visible on the plot of the top panel for each event that could accelerate particles. To indicate the time intervals when we would expect this to occur for each of the events, we use the onset time for each of the EUV waves of Table 1 as the earlier particle release time at the Sun and a travel path length of $0.168 \mathrm{au}$, that is the length of the nominal Parker spiral at perihelion for a solar wind speed of $350 \mathrm{~km} \mathrm{~s}^{-1}$. We overplot the result in the bottom panel of Figure 10. 
Only event \#5-6 can be connected with a clear particle injection with dispersion (the SEP event of April 2). For other events there is no evidence for separate SEP injections. Near the onset of events \#3 and \#9 there are some faint signatures of distinct weak increases of the SEP intensities and also a brief (few hour) intensity increase by about a factor of 3 near 00:00 UT on April 3; this is more apparent in the time profiles of the lower panel. In any case the increases are confined to ions with the lowest energy; they do not seem to show any clear velocity dispersion and it is possible that those small variations in intensity and the $\times 1000$ increase above background of the April 2 event in EPI-Lo are just coincidental with the two events. In particular, the feature near 00:00 UT on April 3 marks a softening of the spectrum of the energetic ion population on those flux tubes, and it does not represent any additional impulsive injection related to coronal activity. Such spectral changes in adjacent flux tubes are not uncommon during SEP events but are of secondary importance to the corona/CME effects that we are highlighting in this study. For event \#13 the changes in the PSP's magnetic connection from April 2 to 4 could be one reason why no SEPs are measured by PSP for this event (see Section 4.1); however, the modeling shows that a shock wave could not form during this eruption. For events \#2 and \#8 the modeling showed that $V_{\mathrm{sh}}^{\prime}$ is below the $V_{\mathrm{A}}$ threshold and shock formation was not possible. Similarly, for events \#1 and \#3 the wave speed was close to the threshold, therefore for these cases a shock may fail to form. No SEP injection was found for those events so the modeling is consistent with the observations.

We conclude that the simple modeling of the events presented in Section 4.3 can adequately explain the SEP observations in all but one case. The only exception is event \#9, for which the modeling suggested shock formation but no SEP was detected by PSP. Comparing this event with event \#5-6, where the wave speed is almost the same and the connectivity should be similar since the two events occurred a few hours apart, there is only one difference that we could find in AIA observations. For event \#5-6 there is a signature of the wave in AIA images on the east limb, whereas for wave \#9 there is not.

\section{The Puzzling Origin of the April 4 SEP Event}

The origin of the SEP event on April 4 proved to be a puzzling case (Leske et al. 2020). Surges have been present in the active region near the onset of the SEP event but no EUV waves are observed in association with these events. Additionally, modeling of PSP's magnetic connectivity shows that a magnetic connection to AR 12738 located nearly $80^{\circ}$ east of the nominal PSP magnetic footpoint was unlikely. There is one eruption that can be connected to the SEP event observed on April 4.

In Section 4.1 we show that a slow SBO-CME enters the COR2 FOV at 22:06 UT on April 2 and evolves through the end of April 4. Two faint "thermal waves" are also observed as a response of the lower atmosphere to the SBO-CME. The first wave appears to spread northward from AR 12738 from 03:00 UT on April 3 and the second spreads parallel to the equator from approximately 08:00 UT on the same day (see Figure 5). Around the onset time of the April 4 SEP event the first wave reaches the northern coronal hole and the second wave extends toward the nominal PSP magnetic footpoints (see Figure 6).

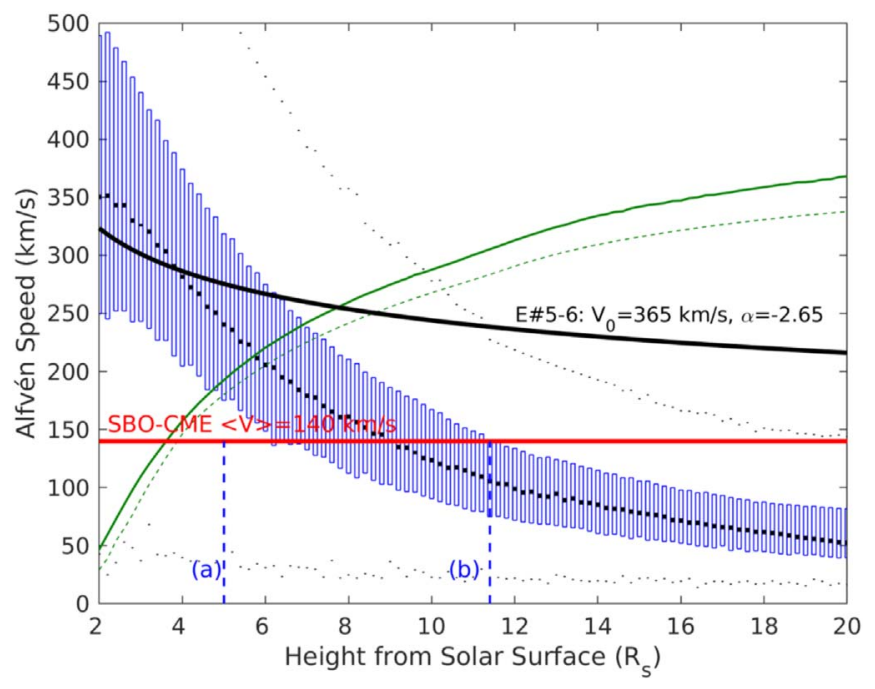

Figure 11. Boxplot representation of the Alfvén speed distributions at the PSPconnected field lines from MAST, using a bin size of $0.2 R_{\odot}$, from $2 R_{\odot}$ to $20 R_{\odot}$. The blue bars represent the first and third quartiles of the distributions, the black lines in the middle show the median values and the extreme black lines show the minimum/maximum values of the distributions defined here as the first/last decile values respectively. The horizontal red line shows the average expansion speed of the SBO-CME. The green lines show the median (solid line) and minimum (dashed line) values of the solar wind speed along the same field lines. Lines (a) and (b) mark the height where the SBO-CME is located near the start and end of the April 4 SEP event.

The SBO-CME expands very slowly with an average speed of $140 \mathrm{~km} \mathrm{~s}^{-1}$. Leske et al. (2020) showed that the differential energy spectrum averaged over the April 4 SEP event was very soft, with an index of -4.36 on energy ( -10.7 on momentum). So if there was a shock produced by the expansion of this SBOCME it must have been very weak, with a compression ratio of 1.4. For a shock normal oblique to the incident magnetic field ( i.e., $\theta_{\mathrm{BN}}=45^{\circ}$ ), this would be associated with a Mach number of about 1.3 (for plasma $\beta \leqslant 0.01$ ).

We use a similar model to that of Section 4.3 to determine whether a shock wave could have formed during the SBOCME eruption. We calculated the Alfvén speed along the PSPconnected magnetic field lines focusing on a $5^{\circ}$ region around the nominal PSP-connection point. We determine the distribution of $V_{\mathrm{A}}$ in discrete bins of height above the solar surface. We show the result of this analysis in Figure 11 using a boxplot representation. We also show in Figure 11 the median (green solid line) and the minimum (green dashed line) values of the solar wind speed along the same field lines. For the SBO-CME propagation speed we use a mean value of $140 \mathrm{~km} \mathrm{~s}^{-1}$ assuming a self-similar expansion. This speed was determined in the plane of the sky of the STA COR2 coronagraph.

This propagation speed of the SBO-CME was higher than the minimum Alfvén speed at the PSP-connected magnetic field lines. This suggests that the SBO-CME could be superAlfvénic and drive a shock wave in specific regions where the local Alfvén speed is a minimum. To further quantify this we calculate the Alfvénic Mach number before and during the SEP event. The SBO-CME during the SEP event was located at a height of $\sim 5 R_{\odot}$ to $\sim 11.5 R_{\odot}$. We mark this interval in Figure 11 with the two vertical blue lines. For the heights we are interested in we have to include the contribution of the solar wind speed in the calculation of the Alfvénic Mach number (see Equation (1)). The shock normal direction $(\hat{\boldsymbol{n}})$ is the only unknown and free parameter in the calculation of $M_{\mathrm{A}}$ from 
Equation (1). We expect that the flanks of the SBO-CME initially expand through the PSP-connected region with $\theta_{\mathrm{VN}}$ (the obliquity of the flow velocity with respect to the shock normal) close to quasi-perpendicular values, and progressively $\theta_{\mathrm{VN}}$ should change toward oblique values.

We continue with the estimation of $\left\langle M_{\mathrm{A}}\right\rangle$ at different stages of the event. Since $\theta_{\mathrm{VN}}$ is unknown, we examine the values that can explain the SEP observations and finally conclude whether those are realistic or not. Just before the start of the April 4 SEP event when the SBO-CME was located around 3 to $5 R_{\odot}$ we found that $\left\langle M_{\mathrm{A}}\right\rangle$ ranges from $\sim 1.0$ to 1.33 for $\theta_{\mathrm{VN}}$ from $75^{\circ}$ to $90^{\circ}$, respectively. At this time interval it is not clear whether a magnetic connection between the SBO-CME and PSP was established or not. During the SEP event (between (a) and (b) in Figure 11) we find that $\left\langle M_{\mathrm{A}}\right\rangle \sim 1.3$ for $\theta_{\mathrm{VN}} \approx 74^{\circ}$, while $\left\langle M_{\mathrm{A}}\right\rangle<1.0$ for $\theta_{\mathrm{VN}}<70^{\circ}$ and $\left\langle M_{\mathrm{A}}\right\rangle \sim 1.9$ for $\theta_{\mathrm{VN}}>80^{\circ}$. After the apparent end of the SEP event our analysis suggests that the SBO-CME should have remained magnetically connected to PSP but no shock existed anymore. For this interval $\left\langle M_{\mathrm{A}}\right\rangle \ll 1$.0for $\theta_{\mathrm{VN}}<70^{\circ}$.

Summarizing the result of this analysis. we find that the SBO-CME could have possibly driven a shock wave. Shock solutions are possible $\left(\left\langle M_{\mathrm{A}}\right\rangle>1\right)$ at PSP-connected field lines during the SEP event for $\theta_{\mathrm{VN}}>70^{\circ}$. Specifically, we find that during the SEP event $\theta_{\mathrm{VN}}$ was about $74^{\circ}$ and $\left\langle M_{\mathrm{A}}\right\rangle \sim 1.3$. Hence a shock wave may have formed with $\langle X\rangle \sim 1.4$. This is consistent with the observed characteristics of the SEP event. A change of $\theta_{\mathrm{VN}}$ to values lower than $70^{\circ}$ near the end of the SEP event results in $\left\langle M_{\mathrm{A}}\right\rangle<1$ and a shock solution is not possible after that time.

\section{Discussion and Conclusions}

A detailed investigation of the solar origin of the SEP events measured by PSP on April 2 and 4 was performed. The EUV and WL observations show that multiple ejections occurred in AR 12738 throughout the PSP passage between April 2 and 4. Most of the eruptions were accompanied by EUV waves. The April 2 SEP event evidently originated from the two homologous ejections that included two surges occurring in quick succession around 08:50 and 09:10, and was accompanied by EUV waves (E. C. Roelof et al. 2020, in preparation). For this event (\#5-6) we performed a detailed 3D modeling in Section 4.2 and we showed that a shock formed during the eruption that was also magnetically connected to PSP.

The modeling of event \#5-6 suggests that the merged EUV wave was fast enough to form a shock in the low corona at the right time to explain the SEP event measured at PSP on April 2 (Figures 7 and 8). The time that the shock wave connects to PSP seems to be highly dependent on the connectivity model used; however, the release time of the SEP event around 09:00 UT is reasonably close to the time that the shock wave connects to PSP at around 09:05 UT. From the wave properties derived by the $3 \mathrm{D}$ modeling and considering the magnetic connectivity, we find that a shock with $\langle X\rangle=1.52$ $\left(\left\langle M_{\mathrm{A}}\right\rangle=1.99\right)$ was responsible for the April 2 SEP event.

The mean shock compression ratio seems to be $\sim 0.15$ larger to explain the observed spectra throughout the event. Only near the end of our modeling interval is $\langle X\rangle \approx 1.38$ for the MAST$\mathrm{FLT}\left(5^{\circ}\right)$ and that gives a spectral index comparable to the observations. We note, however, that when the weak shock wave is closer to the Sun, the spectrum in that region would be likely different than the averaged spectrum that is observed locally at PSP. This depends, however, on the transport properties of the particles.

On April 2 there are no other separate SEP injections detected at PSP that could be associated with any of the other events. In Section 4.3 we examined the likelihood of shock formation during wave \#5-6 by considering a simple model that accounts for the Alfvén speed along the field lines connected to PSP. One other event (\#9) on April 2 has an average speed that exceeds the minimum Alfvén speed registered at the PSP-connected field lines (Figure 9). For this event the observations suggest that the wave could have damped quickly and a shock may never have passed by the PSP-connected field lines. We show that it is unlikely that shocks have formed during the other eruptions on April 2; this is consistent with the PSP SEP measurements.

There is one problem that is difficult to resolve from the modeling. The SEP event continued for many hours $(\sim 23 \mathrm{hr}$, from 09:00 April 2 to 08:00 April 3) after the onset of the event \#5-6, until April 3 when there is an abrupt drop of the SEP intensities when PSP was super-corotating and appears to have crossed a magnetic separatrix region (see E. C. Roelof et al. 2020, in preparation). The very strong (20:1) field-aligned EPILo anisotropies in the energetic He ions unambiguously imply that the acceleration of the $\mathrm{He}$ ions had to continue from the same process for almost $24 \mathrm{hr}$. Since no other eruptions after event \#5-6 could produce SEPs and also no separate SEP injections are actually observed, only event \#5-6 could have a role throughout the SEP event. The wave of event \#5-6 was fast enough to form a shock, but it is unclear for how long the wave continued as a shock wave before it degenerated. We could model the wave until 09:30 UT but after that time we have no information on the evolution of this wave.

In WL STA and LASCO images there are some faint signatures of density structures around the expected time of passage of the wave that can be traced out to $\sim 10 R_{\odot}(16: 00$ April 2). Since the shock wave is likely to have evolved as a blast wave and therefore decelerated significantly, we have that the speed at $\sim 10 R_{\odot}$ is $\sim 244 \mathrm{~km} \mathrm{~s}^{-1}$ (about 1:1.5 of the average speed of Table 1) for a radial density falloff $\rho \propto r^{\alpha}$, where $\alpha=-2.65$. In Figure 11 we show the evolution of the wave speed of event \#5-6 from the blast wave model $\left(V_{\mathrm{sh}} \propto r^{-(\alpha+3) / 2}\right)$. Following the arguments presented in Section 5 for the formation of a shock by the SBO-CME, we find that in the case of the \#5-6 wave a shock could have existed at heights around $20 R_{\odot}$ (e.g., $\sim 18 \mathrm{hr}$ after the onset) for $\theta_{\mathrm{VN}} \geqslant 60^{\circ}\left(\theta_{\mathrm{VN}} \geqslant 40^{\circ}\right.$ for a height around $\left.10 R_{\odot}\right)$. From the propagation direction of the \#5-6 wave those $\theta_{\mathrm{VN}}$ values may not be unexpected, but it is difficult to conclude whether these conditions could actually be met throughout the event. We also note that the modeling of the CME on April 2 (see Appendix) shows that a contribution to the SEPs is not very likely. Even if the CME flanks extend longitudinally as far as the modeling suggests (see discussion in Appendix) we find that a shock could not form at the CME's eastern flank.

The April 4 SEP event is likely associated with a slow SBOCME that started lifting off one day before the SEP event. The SBO-CME is leading to a reconfiguration of the global magnetic field and this could favor the transport of particles away from the AR to the field lines connecting to PSP. However, as discussed by Leske et al. (2020), the composition of this SEP event does not exhibit the ${ }^{3} \mathrm{He}$ and heavy-ion 
enhancements that are usually found in flare-accelerated material. It is also not clear whether the plasma motion in the surges observed early on April 4 could lead to a sufficient compression to accelerate particles to the observed energies. In terms of impulsiveness and the amount of ejected material, the surges on April 4 are weak compared to the events on April 2.

For this CME it was not possible to self-consistently model the eruption in $3 \mathrm{D}$ so we constructed a simpler model to investigate whether a shock could form. Our analysis suggests that the SBO-CME could drive a weak shock over the SEP event under two conditions, e.g., $\theta_{\mathrm{VN}}>70^{\circ}$ and low plasma beta values $(\beta<1)$. As discussed in Section 5 , it is possible that the CME's western flank had $\theta_{\mathrm{VN}}>70^{\circ}$ and passed on the connected field lines as a quasi-perpendicular shock favoring an increased acceleration rate. We found that $\left\langle M_{\mathrm{A}}\right\rangle \sim 1.3$ and $\langle X\rangle \sim 1.4$ for $\theta_{\mathrm{VN}} \sim 74^{\circ}$. These shock parameters are consistent with the very soft energy spectrum of the April 4 SEP event (an index for LET-A protons of -4.36 on energy). We note that a plasma compression ahead of the SBO-CME could also lead to an acceleration of the energetic particles without it being necessary for a shock to form (e.g., Giacalone et al. 2002; Roelof 2015).

The shock modeling proved to be essential to determine whether shock waves could form just before and during the April 2 and 4 SEP events. Our key findings can be summarized as follows.

1. We show evidence that the SEP event measured at PSP on April 2 could have originated in the homologous ejections from AR 12738 that included two surges and EUV waves occurring in quick succession around 09 UT. The merged EUV wave was fast enough to form a shock in the low corona.

2. We show that the evolution of the shock parameters can explain the characteristics of the SEP event measured at PSP on April 2. The shock wave continued its propagation as a blast wave, continually accelerating particles for many hours.

3. We show that it is unlikely that a shock wave formed during any of the other eruptions on April 2 with one exception. For this latter the modeling shows that a shock wave could have formed, but no separate SEP injections were detected by PSP on April 2. We argue that the shock wave is likely to have degenerated quickly before magnetic connection could be established with PSP.

4. We show that the SEP event at PSP on April 4 was most likely associated with an SBO-CME that started lifting off one day before the event and led to a significant reconfiguration of the magnetic field around AR 12738. The slow SBO-CME possibly may have driven a plasma compression or a weak shock with low Mach number.

However, the small size of the SEP events studied here, the uncertainties in the determination of the waves' kinematics from the limited available EUV and coronagraphic observations, and also the uncertainty of the MHD models because AR 12738 was invisible to Earth for many days, limit us in drawing solid conclusions on some details of the shock formation/evolution and the acceleration process. Other mechanisms could also have involved and contributed to the energization of ions in the two observed SEP events. As PSP gets even closer to the Sun during the next six years and the solar activity will hopefully increase, more strong SEP events will be observed. We expect that the methods of shock modeling and estimation of magnetic connectivity will prove to be essential for the determination of the origin of particle events measured by PSP (and Solar Orbiter) in future observations.

The IRAP team acknowledges support from the French space agency (Centre National des Etudes Spatiales, CNES; https://cnes.fr/fr) that funds activity in the plasma physics data center (Centre de Donnés de la Physique des Plasmas, CDPP; http://cdpp.eu/) and the Multi Experiment Data \& Operation Center (MEDOC; https://idoc.ias.u-psud. $\mathrm{fr} / \mathrm{MEDOC}$ ), and the space weather team in Toulouse (SolarTerrestrial Observations and Modelling Service, STORMS; http://stormsweb.irap.omp.eu/). This includes funding for the data mining tools AMDA (http://amda.cdpp. eu/), CLWEB (clweb.cesr.fr/) and the propagation tool (http://propagationtool.cdpp.eu). A. Kouloumvakos acknowledge financial support from the ANR project SLOW_SOURCE, ANR-17-CE31-0006-01 as well as COROSHOCK, and FP7 HELCATS project https://www. helcats-fp7.eu/ under the FP7 EU contract number 606692. A. Vourlidas was supported by NASA grants 80NSSC19K1261 and NNX16AH70G. Parker Solar Probe was designed, built, and is now operated by the Johns Hopkins Applied Physics Laboratory as part of NASA's Living with a Star (LWS) program (contract NNN06AA01C). Support from the LWS management and technical team has played a critical role in the success of the Parker Solar Probe mission.

\section{Appendix Modeling the CME on April 2}

The CME of April 2, is a weak/slow event and no shock is identified in the coronagraphic images during the eruption. We reconstruct this event using a forward modeling technique presented in Rouillard et al. (2020), which is similar to that of Thernisien et al. (2009). The shape of this narrow CME corresponds to a flux rope in the shape of a bent toroid. The flux-rope geometry we use assumes a constant major radius and varying minor radius such that the legs of the flux rope attached to the Sun have a smaller cross section than the apex. We use this simple geometry to fit the CME shape in coronal observations. The geometrical model of the CME can take any desired orientation in 3D until a good visual fit is obtained with the observations. The fitting of the $\mathrm{CME}$ has been performed using multi-viewpoint observations of STA, SOHO, and WISPR and extends from 4 to 16 solar radii.

From the forward modeling of the CME we find that it roughly propagated along a Carrington longitude of $55^{\circ}$ and latitude of $10^{\circ}$ (direction of the central axis). The latitudinal extent of the $\mathrm{CME}$ is about $10^{\circ}-20^{\circ}$ and the longitudinal is much grater. Specifically, the angular separation between the eastern and western flanks is estimated to be about $80^{\circ}$ at 06:00 UT. The nearly frontal view of the CME provided by LASCO C3 observations effectively constrained part of the eastern flank of the CME. The orientation of the CME flanks is almost horizontal with a small tilt of $5^{\circ}$ measuring anticlockwise. The initial CME speed at the apex of the flux rope was $250 \mathrm{~km} \mathrm{~s}^{-1}$ at 05:56 UT on April 2 and increased to $285 \mathrm{~km} \mathrm{~s}^{-1}$ when it exited the COR-2A field of view at around 14 UT on April 2. In the same interval the expansion speed of 
a)

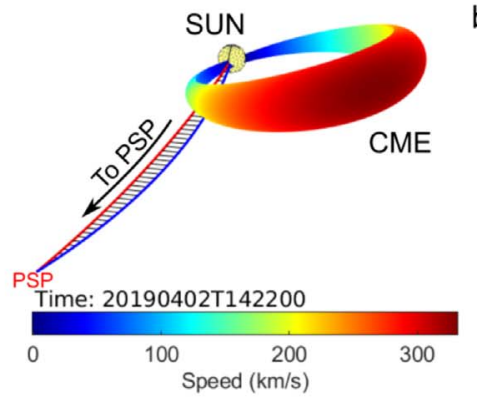

b)

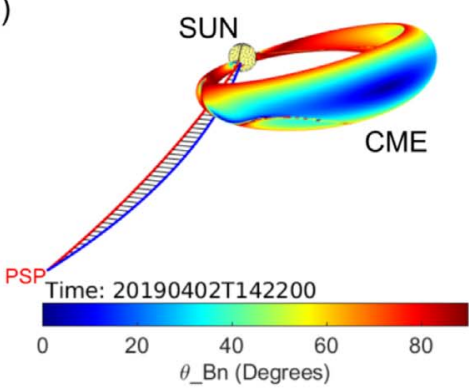

c)

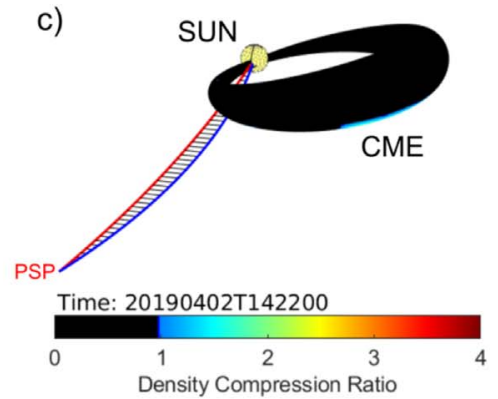

Figure A1. Modeling of the CME on April 2.

the cross section of the flux rope is $40 \mathrm{~km} \mathrm{~s}^{-1}$ and $47 \mathrm{~km} \mathrm{~s}^{-1}$, respectively.

We further model this CME using the methods presented in Section 4.2 but here we adapt our model to a flux-rope shape rather than the ellipsoid used for the EUV wave modeling. We assume that if a shock had formed it occurred close to the surface of the flux rope due to its interaction with the background solar wind. In such a case the resulting sheath region would be weak and narrow. Using the 3D speed of the expanding flux rope and the upstream parameters of the solar wind from the MAST model we computed the shock/pressure wave parameter.

Figure A1 presents different calculated parameters over the CME surface. The Mach number in panel (b) and the density compression ratio in panel (c) are less than unity over most of the CME surface. Overall, the results of this modeling suggest that the CME did not produce a shock throughout its expansion. The modeling also shows that weak shock solutions with Mach numbers greater than unity but below critical values do appear over a narrow but extended region of the flux rope in the orthoradial direction. The subcritical shock in these regions is driven by the self-similar expansion of the flux-rope tube at $\sim 45 \mathrm{~km} \mathrm{~s}^{-1}$. The ability of slow CMEs to compress the background solar wind and produce weak shocks in response to the expansion of the flux rope has been discussed and studied in past papers (e.g., Rouillard et al. 2011; Lugaz et al. 2017). Overall, our modeling shows that the CME is not fast enough to drive a shock wave on any scale.

The modeled CME western flank magnetically connects to PSP during its expansion. We note, however, that the reconstruction technique of Rouillard et al. (2020) assumes a circular current channel for the flux rope, which may lead to an overestimation of the longitudinal extent of the actual CME flanks in this study. Nevertheless, the CME flanks expand very slowly, with a speed of about $185 \mathrm{~km} \mathrm{~s}^{-1}$ at 16 UT. At the well-connected locations we find density compression ratio below 1 (e.g., no shock) and a quasi-perpendicular geometry. The fact that no shock solution was found near those locations is a combination of the low speed and the high $\beta$ values in this region. Note that the condition $M_{\mathrm{A}} \geqslant 1$ does not necessarily guarantee a shock solution in the RH conditions. For an oblique shock wave and for plasma $\beta \sim 1$ we have shock solutions $(X>1)$ for $M_{\mathrm{A}}>1.3$ using the RH jump conditions (see, for example, Figure 9 in Lugaz et al. 2015). A weak shock $(X \sim 1.3)$ could form if the speed of the CME's eastern flank was $50 \mathrm{~km} \mathrm{~s}^{-1}$ greater than our modeled values (e.g., $235 \mathrm{~km} \mathrm{~s}^{-1}$ ). The WL observations do not show such a speed of the CME's flanks. From the CME modeling we conclude that a shock wave is unlikely to have formed during the CME expansion.

\section{ORCID iDs}

Angelos Vourlidas (10 https://orcid.org/0000-0002-8164-5948

Rui Pinto (i) https://orcid.org/0000-0001-8247-7168

Nicolas Poirier (i) https://orcid.org/0000-0002-1814-4673

\section{References}

Bale, S. D., Badman, S. T., Bonnell, J. W., et al. 2019, Natur, 576, 237

Dalla, S., Marsh, M. S., Kelly, J., et al. 2013, JGRA, 118, 5979

Desai, M., \& Giacalone, J. 2016, LRSP, 13, 3

Fox, N. J., Velli, M. C., Bale, S. D., et al. 2016, SSRv, 204, 7

Giacalone, J., \& Jokipii, J. R. 2004, ApJ, 616, 573

Giacalone, J., Jokipii, J. R., \& Kóta, J. 2002, ApJ, 573, 845

Gopalswamy, N., Yashiro, S., Krucker, S., et al. 2004, JGRA, 109, A12105

Howard, R. A., Vourlidas, A., Bothmer, V., et al. 2019, Natur, 576, 232

Kahler, S. W., \& Vourlidas, A. 2013, ApJ, 769, 143

Kaiser, M. L., Kucera, T. A., Davila, J. M., et al. 2008, SSRv, 136, 5 Kouloumvakos, A., Patsourakos, S., Nindos, A., et al. 2016, ApJ, 821, 31 Kouloumvakos, A., Rouillard, A. P., Wu, Y., et al. 2019, ApJ, 876, 80 Kozarev, K. A., Raymond, J. C., Lobzin, V. V., et al. 2015, ApJ, 799, 167 Kwon, R.-Y., \& Vourlidas, A. 2018, JSWSC, 8, A08

Laitinen, T., Dalla, S., \& Marsh, M. S. 2013, ApJL, 773, L29

Lario, D., Kwon, R.-Y., Richardson, I. G., et al. 2017, ApJ, 838, 51

Lario, D., Kwon, R.-Y., Riley, P., et al. 2017, ApJ, 847, 103

Lario, D., Kwon, R.-Y., Vourlidas, A., et al. 2016, ApJ, 819, 72

Leske, R. A., Christian, E. R., Cohen, C. M. S., et al. 2020, ApJS, 246, 35

Lionello, R., Linker, J. A., \& Mikić, Z. 2009, ApJ, 690, 902

Lugaz, N., Farrugia, C. J., Smith, C. W., et al. 2015, JGRA, 120, 2409

Lugaz, N., Farrugia, C. J., Winslow, R. M., et al. 2017, ApJ, 848, 75

Mann, G., Klassen, A., Aurass, H., et al. 2003, A\&A, 400, 329

McComas, D. J., Alexander, N., Angold, N., et al. 2016, SSRv, 204, 187

McComas, D. J., Christian, E. R., Cohen, C. M. S., et al. 2019, Natur, 576, 223

Park, J., Innes, D. E., Bucik, R., et al. 2015, ApJ, 808, 3

Plotnikov, I., Rouillard, A. P., \& Share, G. H. 2017, A\&A, 608, A43

Reames, D. V. 2017, Solar Energetic Particles: A Modern Primer on Understanding Sources, Acceleration and Propagation, Vol. 932 (Berlin: Springer)

Riley, P., Lionello, R., Linker, J. A., et al. 2011, SoPh, 274, 361

Robbrecht, E., \& Wang, Y.-M. 2010, ApJL, 720, L88

Roelof, E. C. 2015, JPhCS, 642, 012023

Rouillard, A. P., Kouloumvakos, A., Vourlidas, A., et al. 2020, ApJS, 246, 37

Rouillard, A. P., Plotnikov, I., Pinto, R. F., et al. 2016, ApJ, 833, 45

Rouillard, A. P., Poirier, N., Lavarra, M., et al. 2020, ApJS, 246, 72

Rouillard, A. P., Sheeley, N. R., Cooper, T. J., et al. 2011, ApJ, 734, 7

Rouillard, A. P., Sheeley, N. R., Tylka, A., et al. 2012, ApJ, 752, 44

Schwadron, N. A., Bale, S., Bonnell, J., et al. 2020, ApJS, 246, 33

Thernisien, A., Vourlidas, A., \& Howard, R. A. 2009, SoPh, 256, 111

Vlahos, L., Anastasiadis, A., Papaioannou, A., et al. 2019, RSPTA, 377, 20180095

Vourlidas, A., Balmaceda, L. A., Stenborg, G., et al. 2017, ApJ, 838, 141

Wiedenbeck, M. E., Bučík, R., Mason, G. M., et al. 2020, ApJS, 246, 42 